\title{
A Geometric Method for Estimating the Nominal Cell Range in Cellular Networks
}

\author{
A. J. García $\mathbb{D D}^{1}{ }^{1}$ V. Buenestado $\mathbb{D}^{\circ},{ }^{2}$ M. Toril $\mathbb{D}^{1},{ }^{1}$ S. Luna-Ramírez $\mathbb{D}^{1},{ }^{1}$ and J. M. Ruiz ${ }^{2}$ \\ ${ }^{1}$ Departamento de Ingeniería de Comunicaciones, Universidad de Málaga, Málaga, Spain \\ ${ }^{2}$ Ericsson, Málaga, Spain \\ Correspondence should be addressed to A. J. García; ajgp@ic.uma.es
}

Received 4 January 2018; Revised 13 March 2018; Accepted 26 March 2018; Published 2 May 2018

Academic Editor: Ioannis D. Moscholios

Copyright ( 2018 A. J. García et al. This is an open access article distributed under the Creative Commons Attribution License, which permits unrestricted use, distribution, and reproduction in any medium, provided the original work is properly cited.

In cellular networks, cell range is a key parameter for network planning and optimization. With the advent of new radio access technologies, it is not easy to obtain a good estimate of the nominal cell range on a cell-by-cell basis due to the complexity of physical layout in a real network. In this work, a novel geometrical method for estimating the cell range based on Voronoi tessellation is presented. The inputs of the method are site locations, antenna azimuths, and antenna horizontal beamwidths. The method is tested with a real dataset taken from a live LTE network. During assessment, the proposed method is compared with traditional approaches of estimating cell range. Results show that the proposed method improves the accuracy of previous approaches, while still maintaining a low computational complexity.

\section{Introduction}

With the increasing complexity of mobile networks, radio network planning has become a challenging task for mobile operators. The aim of planning is to find a cost-effective deployment solution to offer subscribers the best network performance in terms of coverage, capacity, and connection quality [1-3]. For this purpose, in the initial preplanning stage, the required site density is estimated based on link budgets. Then, in the nominal planning stage, optimal site locations are determined in terms of network coverage and capacity. In the final detailed planning stage, the best site configuration is selected. In all these processes, estimating the nominal (i.e., planned) Cell Range (CR) is a critical task since it influences the number of required base stations, their geographical location, and the optimal antenna settings (e.g., transmit power or tilt angle) $[1,2,4,5]$.

An improper network modeling during the planning stage can lead to suboptimal system performance during network operation. This problem can be solved by improving network models with live measurements (a.k.a. measurement-based replanning) or counteracted by tuning radio network parameters (a.k.a. network optimization). In both cases, an accurate estimation of the nominal cell service areas is critical to obtain good results $[4,6]$. An example of such a need is the automatic method proposed in [7] to detect cells with overshooting problems. In that method, the actual (i.e., measured) cell range, obtained from Time Advance (TA) statistics [8], is compared with the nominal cell range. In such a comparison, any deviation of the nominal cell range causes that a cell is classified as an overshooter or not. Similarly, the planning methods proposed in $[9,10]$ for selecting the best antenna tilt angle based on geometric considerations strongly depend on the nominal CR.

Traditionally, operators use two different approaches to estimate the nominal cell range in mobile networks. The first approach consists of using commercial cellular network planning tools [11], mainly based on static systemlevel simulators that allow analyzing coverage, capacity, and quality of service related issues. One of the key processes performed during a step simulation is the dominance area calculation and, thus, nominal cell range. In this process, the link losses from each base station to each position in its calculation area are estimated by using network configuration parameters for base stations, mobiles stations, and the 
network area and different propagation models that allow to simulate path losses in a real environment [12]. Static simulators obtain an accurate estimation of network parameters but assuming a high computational load, especially for highdensity scenarios. Thus, cellular network planning tools are used, generally, in the initial preplanning stage where time requirements are not so restrictive. Alternatively, a geometric calculation of nominal cell range is also commonly used for operators. Geometric calculation is uniquely based on physical information of base stations (e.g., location of base stations). This approach consists of a simple method that allows to obtain, with a considerable grade of accuracy, an estimate of cell range with a very low computational load by avoiding to use complex tools in its process. Thus, it is an efficient alternative used by operator to be integrated in their network management systems for optimization processes since it is able to be executed over large geographical areas in seconds.

In an ideal cellular network with regular geometry, the geometric CR (i.e., the distance to cell edge) can be estimated analytically by using the inter-site distance (ISD) [13]. Specifically, the CR in a hexagonal grid scenario is ISD $/ \sqrt{3}$ with omnidirectional antennas, and ISD/3 with trisectorized antennas. However, in a live scenario, sites are unevenly distributed to deal with factors such as topography, cost, or availability, causing that cell shapes are irregular [14]. Moreover, new radio access technologies (4G/5G) will result in the deployment of a higher number of small cells, increasing the complexity of physical layout [15]. Thus, the CR cannot be calculated by using the distance to the nearest site.

Current operator practice is to compute the nominal CR of a site by averaging the distance to some of the nearest sites. Then, the number of nearest sites selected is limited in an attempt to avoid considering several rings of adjacent cells. Unfortunately, the best number of nearest sites is difficult to define, as it depends on the specific scenario [16]. For simplicity, such a parameter is set to a fixed value, leading to inaccurate $\mathrm{CR}$ estimates in many cases. To solve these limitations, some authors use Voronoi tessellation $[17,18]$ to define the polygon representing the service (or dominance) area for every site $[14,19-22]$. Such a diagram can then be used to choose the nearest sites more accurately and, consequently, to estimate the ISD [21]. Once the nominal ISD is obtained, CR is estimated as half of the ISD value. The main limitation of this method is that the CR assigned to all cells in a site (i.e., cosited cells) is the same, which is seldom true in the live network. Such inaccuracies can jeopardize the benefits of network planning and optimization methods that rely on CR estimates (e.g., [7]).

In this work, a geometric method to calculate the CR based on Voronoi tessellation is presented. The main novelties are that (a) CR is calculated on a cell (and not on a site) basis, (b) CR depends on the antenna pointing direction of each cell (i.e., azimuth), and (c) the antenna beamwidth value is used to define the cell border (i.e., side of the service area). Thus, a more accurate estimation of the CR is obtained with a low computational cost. The analysis is extended by checking the impact of the proposed method on the performance of the cell overshooting detection algorithm described in [7].
The main contributions of this work are (a) a novel and computationally efficient method to estimate the CR on a percell basis suitable for radio network optimization processes; (b) a comprehensive analysis of the proposed method in a real scenario, showing the limitations of current practice and how these can be solved by the new approach, and (c) an evaluation of the impact of CR estimates on the performance of a classical cell overshooting detection algorithm in a live LTE network.

The rest of the work is organized as follows. Section 2 reviews the current method used to calculate the CR on a site basis. Section 3 describes the method proposed to calculate the CR on a cell basis. Section 4 shows the results obtained by the method in a real scenario. Finally, Section 5 presents the main conclusions of the study.

\section{Calculation of Cell Range on a Site Basis}

In a network consisting of $n$ sites, an estimate of CR can be obtained by calculating the average distance to the $k$ nearest sites, where $k<n$ [21]. Thus, the average ISD from site $s$ to the $k$ nearest sites is defined as

$$
\operatorname{ISD}_{s, k}=\frac{1}{k} \sum_{m=1}^{k} \operatorname{dist}(s, m),
$$

where dist $(s, m)$ is the Euclidean distance from site $s$ to site $m$. Once the average ISD of site $s$ is calculated with the $k$ closest neighbor sites, the CR of cell $c$ located in site $s$ is calculated as

$$
\mathrm{CR}^{\text {site }}(c)=\frac{\mathrm{ISD}_{s, k}}{2}
$$

where $\operatorname{ISD}_{s, k}$ is the ISD of site $s$ calculated by using the $k$ nearest sites. Note that $\mathrm{CR}^{\text {site }}\left(c_{i}\right)=\mathrm{CR}^{\text {site }}\left(c_{j}\right) \forall c_{i}, c_{j} \in s$.

Figure 1 shows an example of how the current method works. $\mathrm{CR}^{\text {site }}$ is represented by a dashed circumference and the pointing direction of cell by a solid arrow. In this example, it is assumed that the number of nearest sites used for ISD calculation (represented by crosses with dotted circles) is 6 , and the solid arrow represents the pointing direction of the cell. From the figure, it is clear that the previous method has several limitations, the foremost of which is the calculation of the ISD at a site level, causing that all cells in the same site are assigned the same CR value (represented by a dashed arrow). Moreover, the CR of a cell is only based on the distance between the site where the cell is located and surrounding sites. However, in a real network, the service area of a cell with directional antennas is mainly determined by the sites that are in the pointing direction of (i.e., in front of) the cell.

\section{Calculation of Cell Range on a Cell Basis}

To solve the above-described problems, the CR should be computed in a cell level making the most of geometric considerations. This is done in the method described next. The method consists of three stages: Voronoi tessellation, cell border definition, and computation of average distance to cell border. 


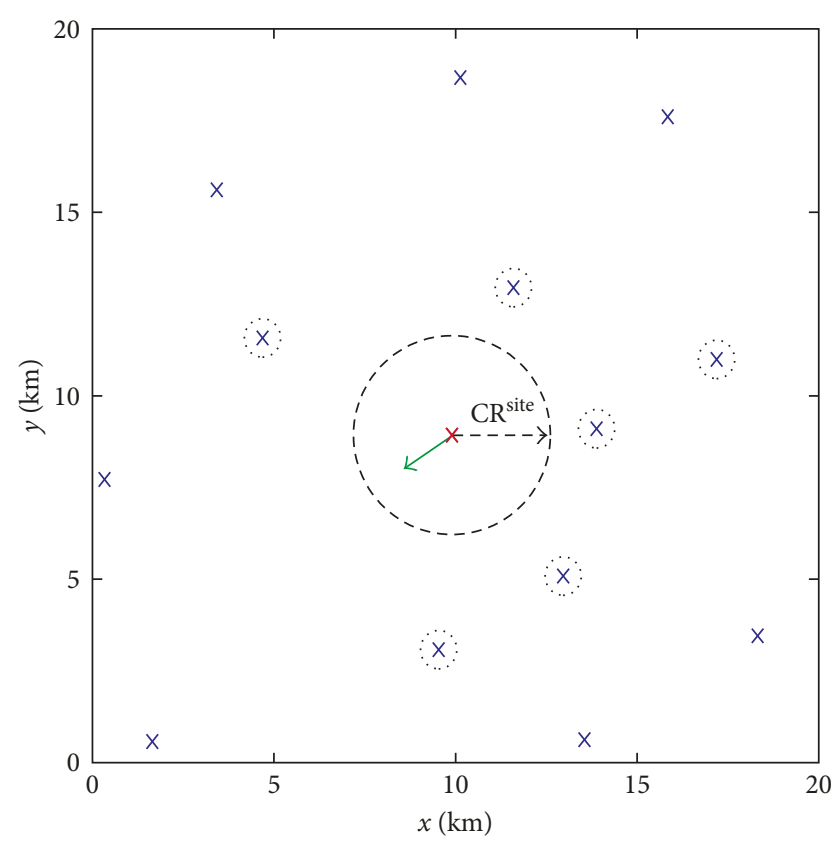

FIGURE 1: Computation of the average inter-site distance on a site basis.

3.1. Voronoi Tessellation. A Voronoi diagram is defined as a partitioning of a plane into regions based on the distance to points in a specific subset of the plane [23]. Let $P=\left\{p_{1}, p_{2}, \ldots, p_{n}\right\}$ be a set of points in the plane, called nodes. $v\left(p_{i}\right)$ is defined as the Voronoi cell of $p_{i}$ formed by the set of points $q$ in the plane that are closer to $p_{i}$ than to other nodes. Thus, the Voronoi cell of node $p_{i}$ is defined as

$$
v\left(p_{i}\right)=\left\{q \mid \operatorname{dist}\left(p_{i}, q\right)<\operatorname{dist}\left(p_{j}, q\right), \forall j \neq i\right\},
$$

where $\operatorname{dist}\left(p_{i}, q\right)$ is the Euclidean distance from node $p_{i}$ to point $q$ and $\operatorname{dist}\left(p_{j}, q\right)$ is the Euclidean distance from node $p_{j}$ to point $q$.

$v\left(p_{i}\right)$ can also be defined as an intersection of planes. Given two nodes, $p_{i}$ and $p_{j}$, the set of points that are strictly closer to $p_{i}$ than $p_{j}$ is the open half plane whose line that separates the two half planes is the perpendicular bisector between $p_{i}$ and $p_{j}$ formed by those points equidistant from these two nodes. This half plane is denoted as $h\left(p_{i}, p_{j}\right)$. Thus, the Voronoi cell of $p_{i}$ is defined as

$$
v\left(p_{i}\right)=\cap_{j \neq i} h\left(p_{i}, p_{j}\right) .
$$

Figure 2 shows an example of Voronoi diagram for a set of nodes taken from a real cellular scenario. Nodes (represented by crosses) in the figure represent the sites of the network. It is observed that each Voronoi cell contains all the nearest points to each node, representing the dominance area of the site.

There are several algorithms to compute the Voronoi diagram. The computational complexity of most of them is $O\left(n^{2}\right)$, where $n$ is the number of nodes. However, some algorithms use more efficient methods that reduce the computational complexity to $O(n \log n)$ [23]. An example of the latter is Fortune's algorithm [23], used in this work.

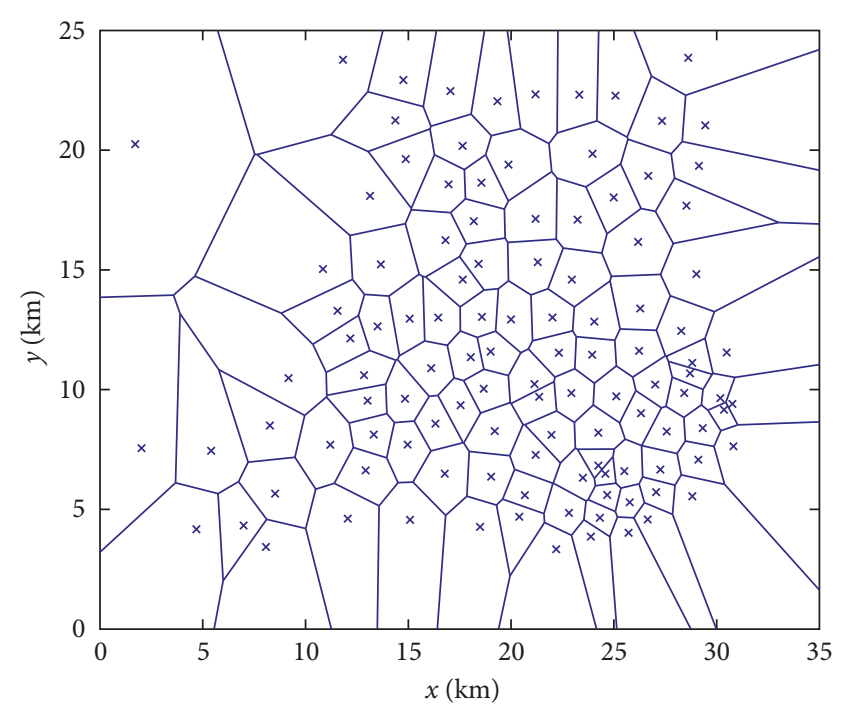

FIgURE 2: Voronoi diagram for a set of sites in a real cellular scenario.



FIGURE 3: Cell border definition.

3.2. Cell Border Definition. Once the Voronoi diagram is constructed for the whole scenario, the next step is to define the cell border from the Voronoi cell generated for each site. Such a cell border is later used to compute the CR (i.e., distance to cell edge).

The process to estimate the cell border is shown in Figure 3. The inputs to the methods are site locations (represented by crosses), polygons defining the Voronoi cells (solid lines), antenna pointing direction in the horizontal plane (arrows), and beamwidth $(\theta)$. All the required data (except Voronoi polygons) are included in network planning files used by the operator. The cell border can be obtained graphically by the intersection between the straight lines defined by the beamwidth (dashed lines) and the outline of the Voronoi cell (solid lines). 
To automate the method, it is necessary to find the line equation of each line segment defining the cell shape (labeled as $L_{1}, L_{2}$, and $L_{3}$ in the figure). For this purpose, the coordinates $(x, y)$ of all intersection points $(\mathrm{A}, \mathrm{B}, \mathrm{C}$, and $\mathrm{D}$, in the figure) can be obtained by matching the equations of the Voronoi cell segments and those defined by the beamwidth. Then, intersection points that are not located in the pointing direction (arrow) are discarded (C and D in Figure 3). Finally, cell border consists of the line segments defined by the nondiscarded intersection points (A and $\mathrm{B}$ ) and the Voronoi cell (solid lines). Note that, depending on the beamwidth value and the shape of the Voronoi cell, the cell border is defined by different line segments.

3.3. Computation of Average Distance to Cell Border. The final step is to obtain the CR value from the cell border. For this purpose, the average distance from the site to all segments defining the cell border is calculated analytically. As the cell border can consist of more than one segment, it is necessary to calculate the average distance from the site where cell is located to each of those segments.

The average distance from a point $P$ to a line segment $L$ can be calculated by a simple transformation in the coordinate system. Figure 4 illustrates that change. In the new coordinate system $\left(X^{\prime} Y^{\prime}\right)$, the line segment under calculation must be located in the positive $x$-axis. In the new coordinate system, the average distance between the point and the line segment, $\overline{\operatorname{dist}}(P, L)$, is defined as

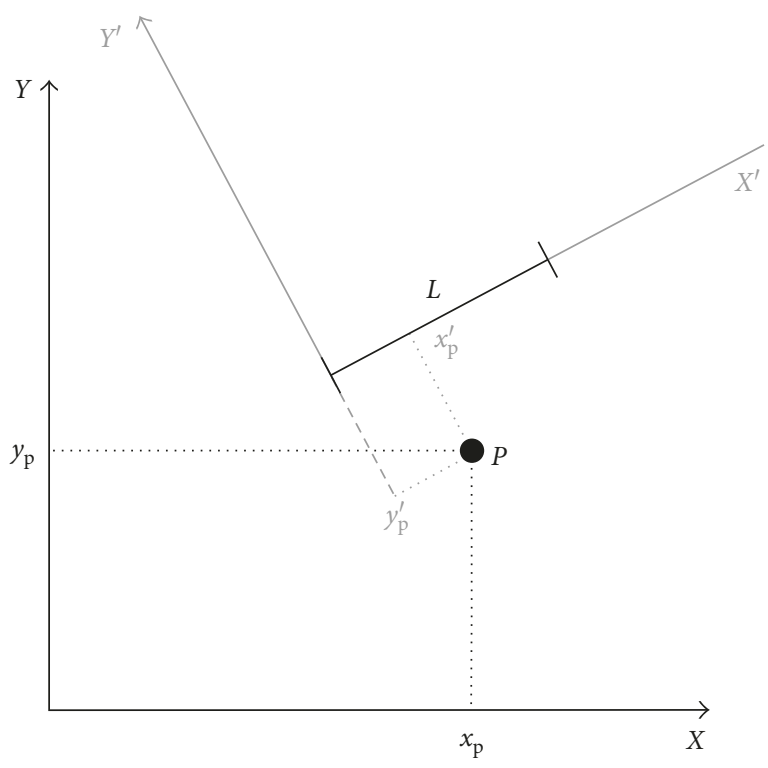

FIgURe 4: Point translation.

$$
\overline{\operatorname{dist}}(P, L)=\frac{1}{l_{L}} \int_{0}^{l_{L}} \sqrt{\left(x^{\prime}-x_{p}^{\prime}\right)^{2}+y_{p}^{\prime 2}} d x^{\prime},
$$

where $\left(x_{p}^{\prime}, y_{p}^{\prime}\right)$ is the point location in the new coordinates system and $l_{L}$ is the length of the line segment $L$.

The integral shown in (5) can be solved analytically as

$$
\begin{aligned}
\int \sqrt{(x-a)^{2}+b^{2}} d x= & \frac{1}{2 \sqrt{a^{2}+b^{2}-2 a x+x^{2}}}\left\{(-a+x)\left(a^{2}+b^{2}-2 a x+x^{2}\right)-b^{2} \sqrt{a^{2}+b^{2}-2 a x+x^{2}}\right. \\
& \left.\cdot \log \left[2\left(a+\sqrt{\left(b^{2}+(a-x)^{2}\right)}-x\right)\right]\right\}
\end{aligned}
$$

where $a$ and $b$ are $x_{p}^{\prime}$ and $y_{p}^{\prime}$, respectively.

Once the average distance between the site and each segment of the cell border is known by using (6), a weighted average distance is obtained based on the length of each segment. Such a weighting operation ensures that the average distance is dominated by longer segments. Thus, the CR of cell $c$ is computed as

$$
\mathrm{CR}^{\text {cell }}(c)=\frac{\sum_{i=1}^{n_{\text {seg }}(c)} \overline{\operatorname{dist}}\left(s, L_{i}\right) \cdot l_{L_{i}}}{\sum_{i=1}^{n_{\text {seg }}(c)} l_{L_{i}}},
$$

where $n_{\text {seg }}(c)$ is the number of segments in the border of cell $c$ and $\overline{\operatorname{dist}}\left(s, L_{i}\right)$ is the average distance from site $s$ to line segment $L_{i}$.

The proposed geometric method has a limitation for cells in sites located at the border of the scenario when there is no other cell in their pointing direction. As observed in Figure 2, the Voronoi cell in these cases is not bounded and extends to infinity in the pointing direction of the cell. Thus, it is impossible to calculate the $\mathrm{CR}$ by using the geometric method, as the segments defining the cell border cannot be identified. In the absence of a better estimate, the CR might be set to the maximum cell radius given by the link budget in that scenario [4]. Note that such an estimate would still be more accurate than assigning the CR of the other cells in the same site, as the service area of border cells tends to be large.

\section{Performance Assessment}

In this section, the above-described methods of computing the CR on a site and cell basis are compared based on the results obtained in a real LTE network. The analysis is first focused on the CR values obtained by each method. Then, the analysis is extended by checking the impact of the proposed methods on the performance of the cell overshooting detection algorithm proposed in [7]. Finally, the computational load of both methods is evaluated.

4.1. Analysis Set-Up. Most of the analysis is done in an urban scenario consisting of 160 LTE cells, distributed in 54 sites. In this area, only a single carrier is deployed. The dataset 
includes (a) the geographical location of all sites in the area, as well as sites in the surroundings to avoid border effects, (b) the pointing direction of each cell (i.e., azimuth), and (c) the number of cells in each site, used to define the antenna beamwidth (e.g., if there are three cells in the same site, the beamwidth value is $360 / 3=120^{\circ}$ ).

To compare methods, the following indicators are calculated:

(1) CR at cell level, $\mathrm{CR}^{\text {cell }}$, which is the indicator proposed in this work, computed as in (7).

(2) $\mathrm{CR}$ at site level, $\mathrm{CR}^{\text {site }}$, which is the indicator used in prior works, computed as in (2) with the $k=6$ nearest sites. The value of 6 sites intends to take into account the first tier of neighbors.

Comparison is done by computing CR relative differences as

$$
\varepsilon^{\text {site,cell }}(c)=\frac{\mathrm{CR}^{\text {site }}(c)-\mathrm{CR}^{\text {cell }}(c)}{\mathrm{CR}^{\text {cell }}(c)}
$$

The cell overshooting detection algorithm presented in [7] is used to check the impact of estimating CRs by different methods (i.e., site level or cell level). In the algorithm, candidate cells for downtilting are ranked based on the comparison of the nominal (planned) and real (measured) cell edge. The actual maximum serving distance of each cell, hereafter referred to as measured cell range, can be derived from TA measurements collected by the base station (eNodeB) [24]. The TA procedure adjusts uplink transmissions to ensure that the downlink and uplink subframes are synchronized at the base station. Thus, the TA value can be directly mapped to the distance between the user and the base station (i.e., eNodeB in LTE). In LTE, TA resolution is $0.52 \mu \mathrm{s}$ $(78 \mathrm{~m})$. A statistical TA distribution for each cell can be generated by collecting TA measurements over long time periods (e.g., one day). From this data, the measured CR, $\mathrm{CR}^{\text {meas }}$, is defined as the 95th percentile of the TA distribution (i.e., the distance exceeded only by $5 \%$ of the users served by the cell). Once the measured and nominal CRs are known, the ratio between both indicators can be used as an indicator of cell overshooting. Specifically, the overshooting ratio (OVSR) is defined as the ratio between the measured and the nominal CRs. A large value of OVSR in a cell indicates that the cell is capturing users farther than planned. Thus, the higher the ratio, the higher the need to increase antenna tilt of the cell.

To measure the computational load, both methods are implemented in Matlab ${ }^{\odot}$. The Voronoi tessellation is computed by the Voronoi routine [25]. Then, the methods are tested in two scenarios, consisting of 160 and 12,500 LTE cells, distributed in 54 and 2966 sites, respectively. All methods are executed in an Intel ${ }^{\odot}$ Core i5 dual-core computer with $2.6 \mathrm{GHz}$ clock frequency and $8 \mathrm{~GB}$ of RAM. In this case, method assessment is done based on the execution time.

\section{Results}

A comparison of methods is first carried out by checking the correlation between the indicators $\mathrm{CR}^{\text {site }}$ and $\mathrm{CR}^{\text {cell }}$. From

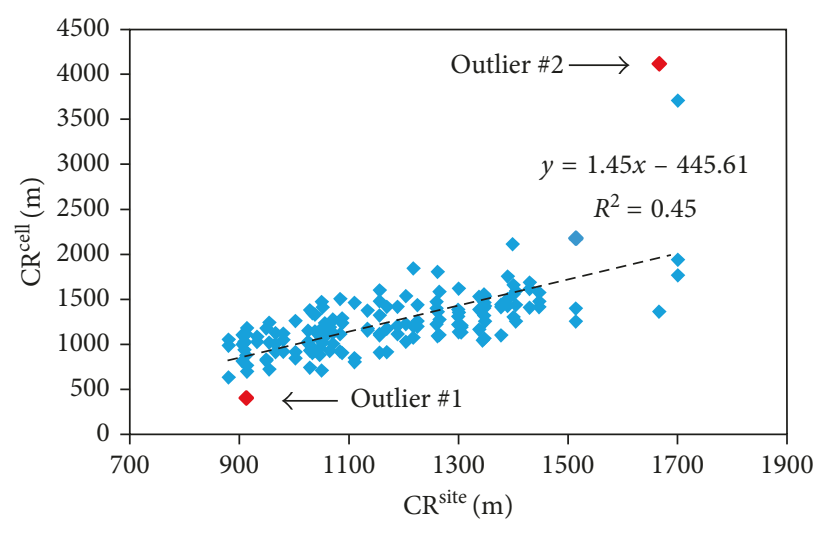

FIgURE 5: Comparison of cell ranges obtained by the site-level and cell-level methods.

this analysis, two abnormal cases are identified and discussed in more detail.

Figure 5 shows the comparison of CR values obtained by the methods. The $x$-axis represents $\mathrm{CR}^{\text {site }}$ values, whereas the $y$-axis represents $\mathrm{CR}^{\text {cell }}$ values. Each point in the figure represents a cell in the scenario. To ease the comparison, regression line is superimposed. It is observed that, even if site-level and cell-level CRs are related, correlation is not strong, which is clear from the modest value of the determination coefficient (i.e., $R^{2}=0.45$ ).

In some cells, the differences between site- and cell-level estimates are noticeable. Specifically, relative differences range from $\varepsilon^{\text {site,cell }}=-61 \%$ (outlier $\# 2$ ) up to $128 \%$ (outlier $\# 1$ ), with $-1.86 \%$ as the average value. The following analysis is restricted to abnormal cases, as the rest of cases show similar results for $\mathrm{CR}^{\text {cell }}$ and $\mathrm{CR}^{\text {site }}$, deduced from the low average value of the relative difference $(-1.86 \%)$. To find the cause of such differences, a closer analysis is carried out on the two extreme cases highlighted in Figure 5.

The first abnormal case (outlier \#1) corresponds to a cell with CR in a cell level much smaller than CR in a site level (i.e., $\mathrm{CR}^{\text {cell }} \ll \mathrm{CR}^{\text {site }}$ ). Figure 6 depicts the local environment of this cell in more detail, showing site locations (crosses), the Voronoi diagram (polygons), the cell azimuth (arrow), the 6 neighbor site locations (crosses with dotted circles), the CR in a site level (dashed arc), and the CR in a cell level (solid arc). As observed in the figure, the analyzed cell has a nearby site just in front of it, but the other 5 nearest sites used to calculate the $\mathrm{CR}^{\text {site }}$ are farther. Thus, $\mathrm{CR}^{\text {site }}$ (dashed circle) is much larger than $\mathrm{CR}^{\text {cell }}$ (solid line).

The other abnormal case (outlier \#2) corresponds to a cell where the CR estimated in a cell level is much larger than in a site level (i.e., $\mathrm{CR}^{\text {site }} \ll \mathrm{CR}^{\text {cell }}$ ). Figure 7 shows the situation where the closest site in front of a cell is much farther than the 6 nearest sites used to compute $\mathrm{CR}^{\text {site }}$. This causes that the CR obtained by averaging ISDs, $\mathrm{CR}^{\text {site }}$ (dashed arc), is smaller than the one obtained from polygons in the Voronoi diagram, $\mathrm{CR}^{\text {cell }}$ (solid arc).

Note that, in both abnormal cases, the correct solution is given by the proposed method, as it can adapt to the local differences between cells of the same site.

Table 1 presents the numerical values of CR obtained in both cases to quantify the difference between methods. From 


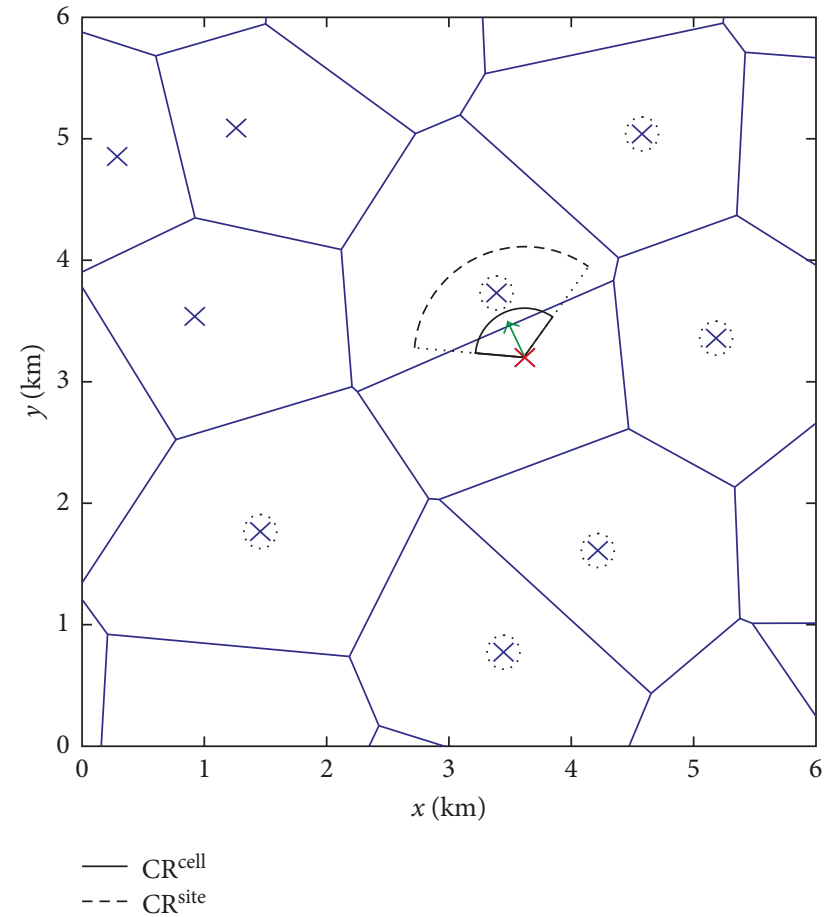

FIgURE 6: Abnormal case 1: a nearby site located in the pointing direction.

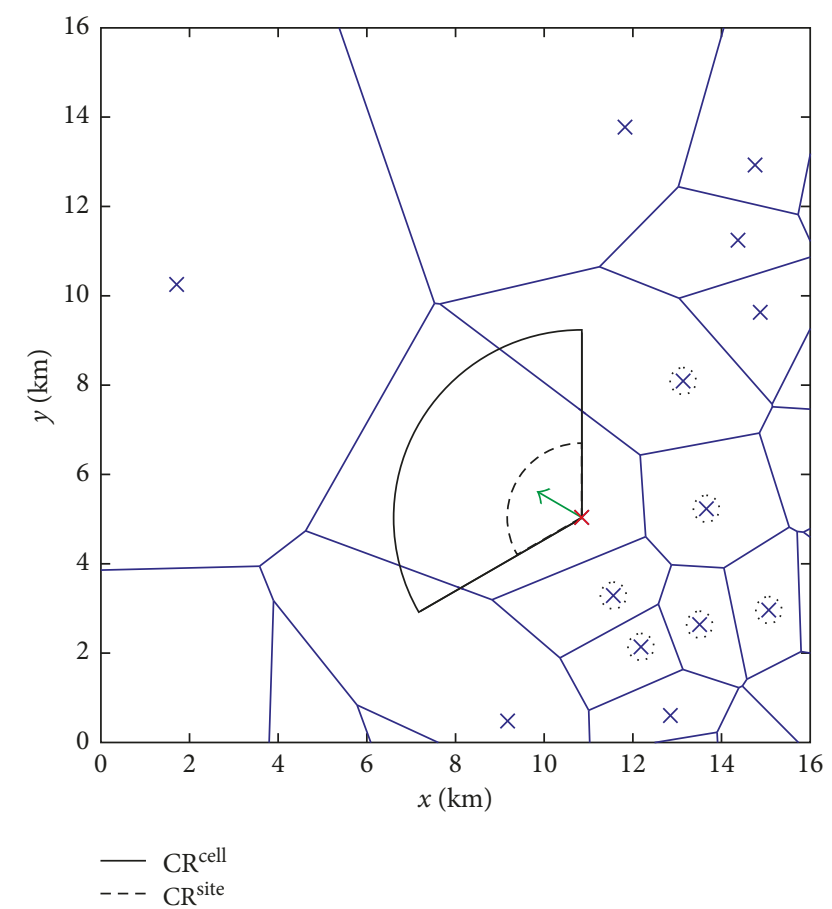

Figure 7: Abnormal case 2: a distant site located in the pointing direction.

the values, it can be verified that CR relative difference in a real environment can be of up to $128 \%$.

Having detected large differences in the CRs obtained by both methods, the following experiment checks the impact
TABLE 1: Comparison of CR estimates.

\begin{tabular}{lcc}
\hline Case & Nearby site & Distant site \\
\hline $\mathrm{CR}^{\text {cell }}(\mathrm{km})$ & 0.40 & 4.10 \\
$\mathrm{CR}^{\text {site }}(\mathrm{km})$ & 0.91 & 1.60 \\
$\varepsilon^{\text {site,cell }}(\%)$ & 128 & -61 \\
\hline
\end{tabular}

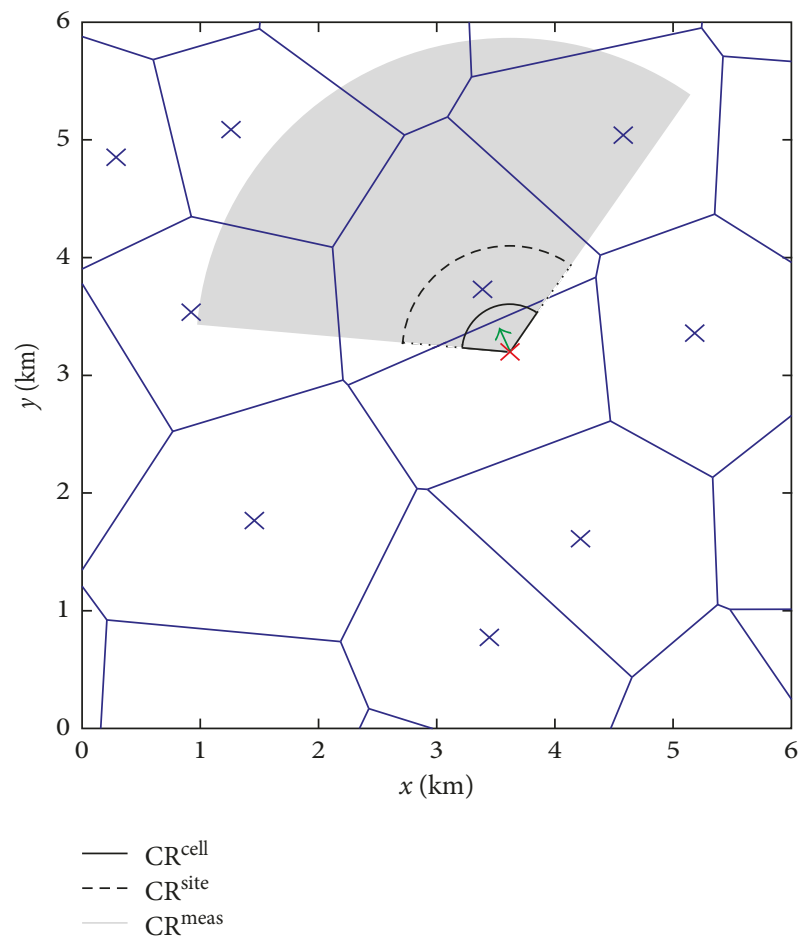

Figure 8: Measured versus nominal cell ranges with a nearby site.

TABLE 2: Measured versus estimated cell ranges.

\begin{tabular}{lcc}
\hline Case & Nearby site & Distant site \\
\hline $\mathrm{CR}^{\text {meas }}(\mathrm{km})$ & 2.67 & 4.03 \\
$\mathrm{CR}^{\text {cell }}(\mathrm{km})$ & 0.40 & 4.10 \\
$\mathrm{CR}^{\text {site }}(\mathrm{km})$ & 0.91 & 1.60 \\
OVSR $^{\text {cell }}$ & 6.68 & 0.98 \\
OVSR $^{\text {site }}$ & 2.93 & 2.52 \\
\hline
\end{tabular}

of each method on the cell overshooting detection algorithm presented in [7]. For brevity, the analysis is restricted to the two cases presented above. The analysis is carried out by computing the overshooting indicator with the nominal CR obtained in a site and a cell level.

Figure 8 represents the case of a nearby site located in front of the cell under study. It is observed that $\mathrm{CR}^{\text {meas }}$ (gray fill) is much larger than both $C R$ estimates $\left(\mathrm{CR}^{\text {cell }}\right.$, represented by a solid arc, and $\mathrm{CR}^{\text {site }}$, represented by a dashed arc). The exact values of the measured CR and OVSR in a site and cell level are shown in Table 2. As observed in Figure 8, the arc defined by the measured CR largely overlaps with the Voronoi cell of the neighbor sites. This is a clear indication 


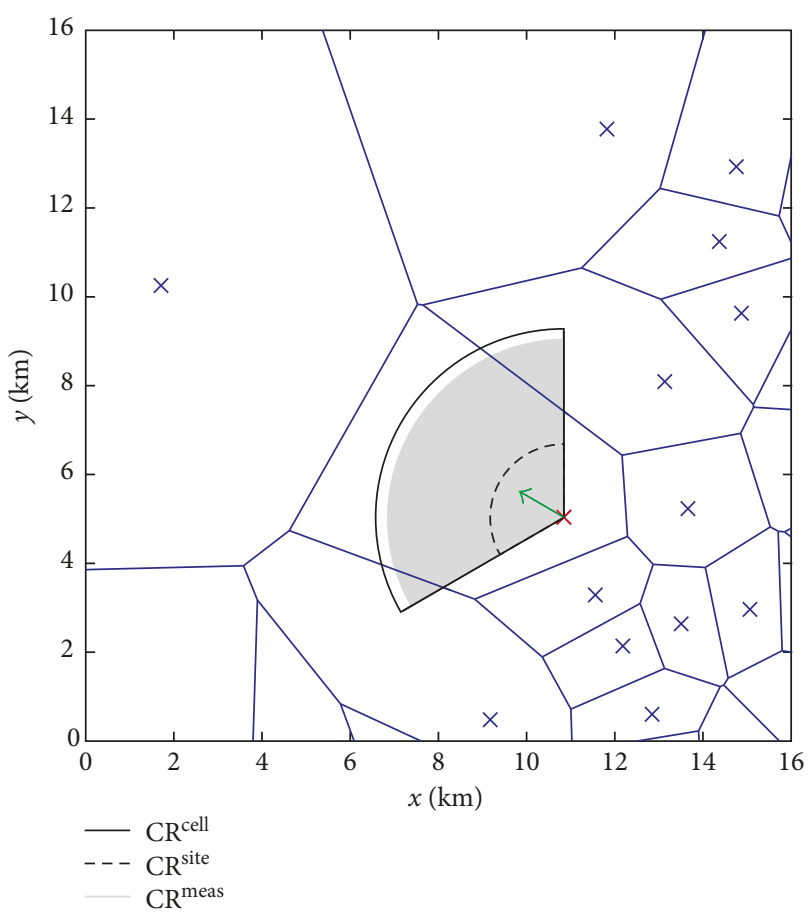

FIGURE 9: Measured versus nominal cell ranges with a distant site.

that the tilting angle of the cell under study is incorrectly planned. Table 2 confirms that, with $\mathrm{CR}^{\text {cell }}$, the cell overshooting indicator has a larger value, and the cell would therefore be prioritized in next replanning actions. In contrast, using $\mathrm{CR}^{\text {site }}$ leads to a lower value of the overshooting indicator, which would be interpreted by the operator as if the tilt angle was not very wrongly configured or even properly set.

Figure 9 shows the other case where the closest site in front of the cell is very distant. In this case, the measured $\mathrm{CR}, \mathrm{CR}^{\text {meas }}$, is similar to the nominal CR in a cell level, $\mathrm{CR}^{\text {cell }}$. Table 2 confirms that the OVSR for the cell-level solution is close to one (i.e., ratio $c_{c}=0.98$ ), showing that the real and nominal CRs in a cell level are pretty close. Thus, no replanning action would be triggered. In contrast, the measured $\mathrm{CR}, \mathrm{CR}^{\text {meas }}$, is much larger than the nominal CR in a site level, $\mathrm{CR}^{\text {site }}$, causing that the OVSR for the site level solution is much larger than 1 (i.e., ratio $_{s}=2.52$ ). This might trigger an unnecessary replanning action.

From these results, it can be concluded that the proposed geometric method for estimating the nominal cell range outperforms the classical approach, since it gives nominal CR estimates closer to those intended in the planning stage.

5.1. Computational Complexity. The two approaches to compute the nominal CR are tested in two scenarios: the urban scenario comprising 160 cells (referred to as S1) and a larger scenario consisting of 12,500 cells (referred to as S2), covering $131.000 \mathrm{~km}^{2}$ with very different environments (rural, urban, and dense urban). Table 3 shows the time required to calculate $\mathrm{CR}^{\text {cell }}$ and $\mathrm{CR}^{\text {site }}$ in both scenarios. It is observed that, in the small scenario, both methods have a similar execution
TABLE 3: Execution time.

\begin{tabular}{lcc}
\hline & $\mathrm{S} 1$ & $\mathrm{~S} 2$ \\
\hline $\mathrm{CR}^{\text {cell }}$ time (s) & 0.60 & 7.10 \\
$\mathrm{CR}^{\text {site }}$ time (s) & 0.50 & 22.5 \\
\hline
\end{tabular}

time. However, in the large scenario, the proposed geometrical method takes one-third of the time needed for the approach based on averaging ISDs. Specifically, in the large scenario, $\mathrm{CR}^{\text {cell }}$ time is $7.1 \mathrm{~s}$ whereas $\mathrm{CR}^{\text {site }}$ time $22.5 \mathrm{~s}$. This is explained by the computational complexity of the methods. The complexity the method based on average ISDs is $O\left(n^{2}\right)$, where $n$ is the total number of sites, as it has to compute the distance between every pair of sites to identify the 6 nearest sites. In contrast, the complexity of the geometric method based on Fortunes algorithm is $O(n \log n)$. These results show that the proposed method is the best option for large scenarios.

\section{Conclusions}

In this work, a geometric method for estimating the nominal cell range on a cell-by-cell basis in a cellular network has been described. The inputs of the method are common planning data, such as site locations, antenna azimuths, and antenna horizontal beamwidths. The method has been tested with a real dataset taken from a live LTE network. During assessment, the proposed method has been compared with the classical approach of estimating cell range, based on averaging the inter-site distance. Results have shown that, in a real scenario, relative difference in the nominal CR estimates obtained by the methods can be of up to $128 \%$. A visual inspection of the results in specific cases has shown that the proposed geometric method leads to more realistic CR values. This is mainly due to its capability to consider local differences between sectors of the same site. Then, CR estimates have been used to build a cell overshooting indicator for each cell as in [7]. Results have shown that the classical approach fails to detect overshooting cells in some cases. The proposed method is conceived to be integrated in network planning and optimization suites running in the network management system. Unlike other approaches based on propagation predictions, it is easy to develop, as there are very effective codes in the public domain implementing Fortunes algorithm. Execution times are low enough to obtain nominal cell ranges of large geographical areas in seconds. It can also be used to obtain the nominal cell range of a newly added cell and update that of surrounding cells. The method can be applied to any radio access technology. Likewise, it can be extended to multilayered heterogeneous scenarios, provided that a separate Voronoi diagram is built for each network layer.

\section{Conflicts of Interest}

The authors declare that they have no conflicts of interest.

\section{Acknowledgments}

This work has been funded by the Spanish Ministry of Economy and Competitiveness (TIN2012-36455) and 
Optimi-Ericsson and Agencia IDEA (Consejería de Ciencia, Innovación y Empresa, Junta de Andalucía, ref. 59288), cofunded by FEDER.

\section{References}

[1] J. Laiho, A. Wacker, and T. Novosad, Radio Network Planning and Optimisation for UMTS, John Wiley \& Sons, Hoboken, NJ, USA, 2006.

[2] A. R. Mishra, Fundamentals of Cellular Network Planning and Optimisation: $2 G / 2.5 G / 3 G$. . Evolution to $4 G$, John Wiley \& Sons, Hoboken, NJ, USA, 2004.

[3] E. Amaldi, A. Capone, and F. Malucelli, "Radio planning and coverage optimization of $3 \mathrm{G}$ cellular networks," Wireless Networks, vol. 14, no. 4, pp. 435-447, 2008.

[4] A. R. Mishra, Advanced Cellular Network Planning and Optimisation: $2 G / 2.5 G / 3 G$... Evolution to $4 G$, John Wiley \& Sons, Hoboken, NJ, USA, 2007.

[5] A. Simonsson, M. Johansson, and M. Lundevall, "Antenna and propagation parameters modeling live networks," in Proceedings of the Vehicular Technology Conference (VTC Fall), pp. 1-5, San Francisco, CA, USA, September 2011.

[6] J. Ramiro and K. Hamied, Self-Organizing Networks (SON): Self-Planning, Self-Optimization and Self-Healing for GSM, UMTS and LTE, John Wiley \& Sons, Hoboken, NJ, USA, 2011.

[7] V. Wille, M. Toril, and R. Barco, "Impact of antenna downtilting on network performance in GERAN systems," IEEE Communications Letters, vol. 9, no. 7, pp. 598-600, 2005.

[8] T. Halonen, J. Romero, and J. Melero, GSM, GPRS and EDGE Performance: Evolution Towards 3G/UMTS, John Wiley \& Sons, Hoboken, NJ, USA, 2004.

[9] W. Jianhui and Y. Dongfeng, "Antenna downtilt performance in urban environments," in Proceedings of the Military Communications Conference (MILCOM'96), vol. 3, pp. 739744, Reston, VA, USA, October 1996.

[10] J. Niemelä, T. Isotalo, and J. Lempiäinen, "Optimum antenna downtilt angles for macrocellular WCDMA network," EURASIP Journal on Wireless Communications and Networking, vol. 2005, no. 5, p. 610942, 2005.

[11] J. Lempiäinen and M. Manninen, Radio Interface System Planning for GSM/GPRS/UMTS, Springer Science \& Business Media, Berlin, Germany, 2007.

[12] A. Wacker, J. Laiho-Steffens, K. Sipila, and M. Jasberg, "Static simulator for studying WCDMA radio network planning issues," in Proceedings of the 49th Vehicular Technology Conference, vol. 3, pp. 2436-2440, Houston, TX, USA, May 1999.

[13] P. M. Shankar, Introduction to Wireless Systems, Wiley, New York, NY, USA, 2002.

[14] A. E. Baert and D. Seme, "Voronoi mobile cellular networks: topological properties," in Proceedings of the Third International Symposium on Algorithms, Models and Tools for Parallel Computing on Heterogeneous Networks, pp. 29-35, Vancouver, Canada, July 2004.

[15] J. An, K. Yang, J. Wu, N. Ye, S. Guo, and Z. Liao, “Achieving sustainable ultra-dense heterogeneous networks for 5G," IEEE Communications Magazine, vol. 55, no. 12, pp. 84-90, 2017.

[16] M. Toril, V. Wille, and R. Barco, "Identification of missing neighbor cells in GERAN," Wireless Networks, vol. 15, no. 7, pp. 887-899, 2009.

[17] M. De Berg, O. Cheong, M. Van Kreveld, and M. Overmars, Computational Geometry: Introduction, Springer, Berlin, Germany, 2008.
[18] F. Aurenhammer, "Voronoi diagrams—a survey of a fundamental geometric data structure," ACM Computing Surveys, vol. 23, no. 3, pp. 345-405, 1991.

[19] M. Sengoku, H. Tamura, S. Shinoda, and T. Abe, "Graph \& network theory and cellular mobile communications," in Proceedings of the 1993 IEEE International Symposium on Circuits and Systems (ISCAS'93), pp. 2208-2211, Chicago, IL, USA, May 1993.

[20] K. Guruprasad, "Generalized Voronoi partition: a new tool for optimal placement of base stations," in Proceedings of the 5th International Conference on Advanced Networks and Telecommunication Systems (ANTS), pp. 1-3, Bangalore, India, December 2011.

[21] A. Landström, H. Jonsson, and A. Simonsson, "Voronoibased ISD and site density characteristics for mobile networks," in Proceedings of the Vehicular Technology Conference (VTC Fall), pp. 1-5, Québec City, Canada, September 2012.

[22] S. Luna-Ramírez, M. Toril, M. Fernández-Navarro, and V. Wille, "Optimal traffic sharing in GERAN," Wireless Personal Communications, vol. 57, no. 4, pp. 553-574, 2011.

[23] S. Fortune, "A sweepline algorithm for Voronoi diagrams," Algorithmica, vol. 2, no. 1-4, p. 153, 1987.

[24] S. Sesia, M. Baker, and I. Toufik, LTE-The UMTS Long Term Evolution: From Theory to Practice, John Wiley \& Sons, Hoboken, NJ, USA, 2011.

[25] http://es.mathworks.com/help/matlab/ref/voronoi.html, 2017. 




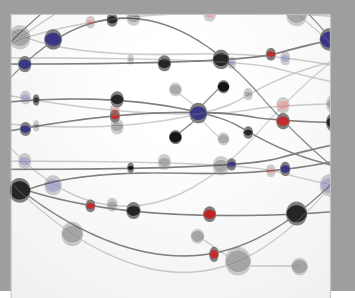

The Scientific World Journal
Submit your manuscripts at

Computing
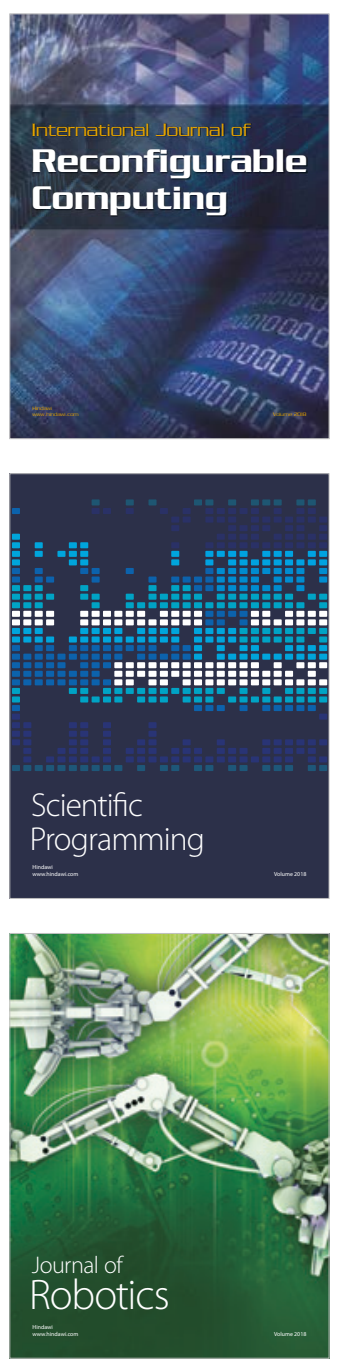

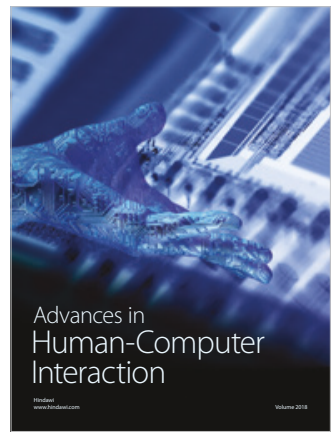

Human-Compute



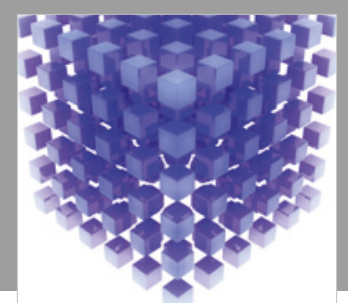

Mathematical Problems in Engineering

\section{Engincering}
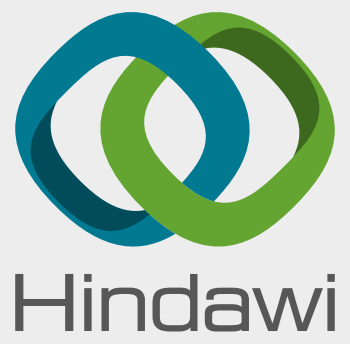

www.hindawi.com
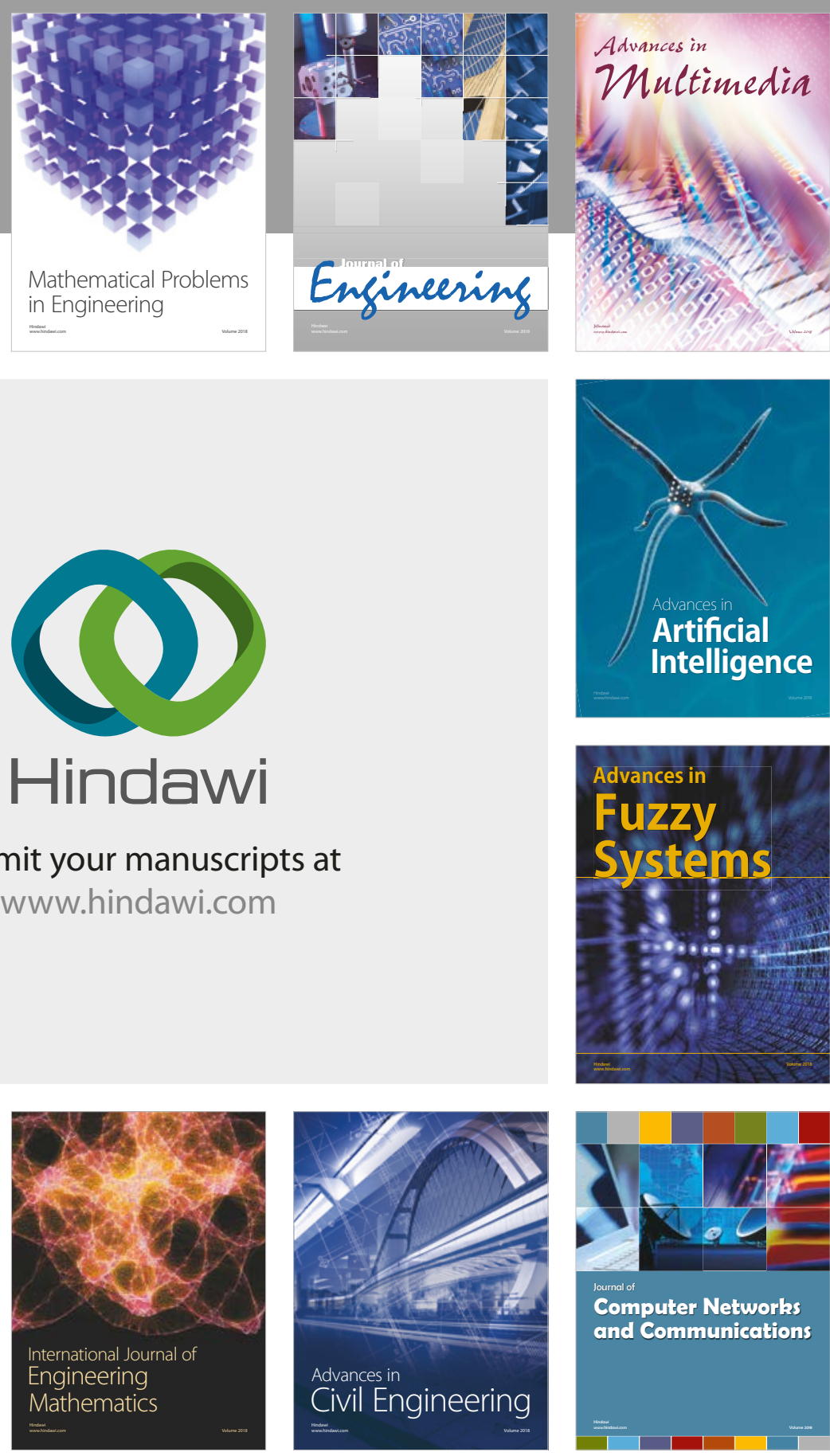

Computer Networks and Communications

Multimedia
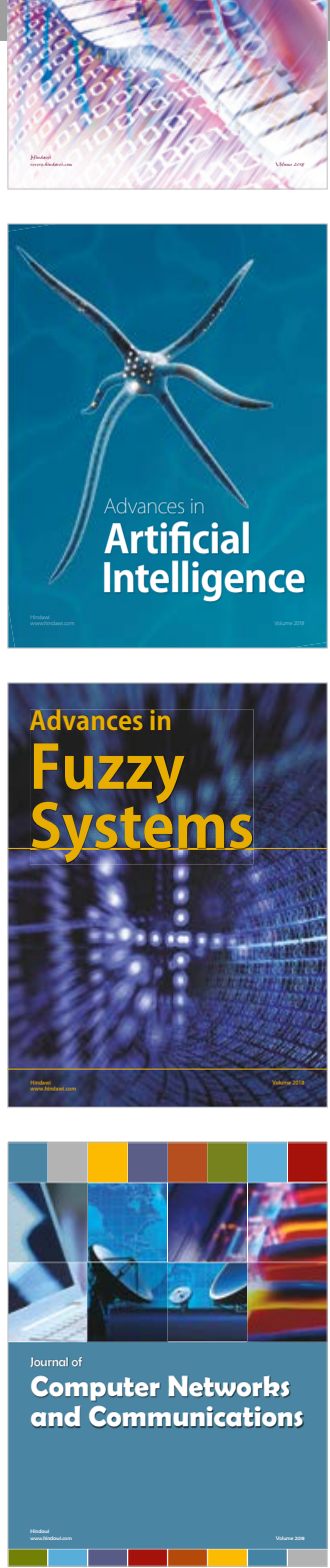

Advances in

Modelling \&

Simulation

in Engineering

interaction



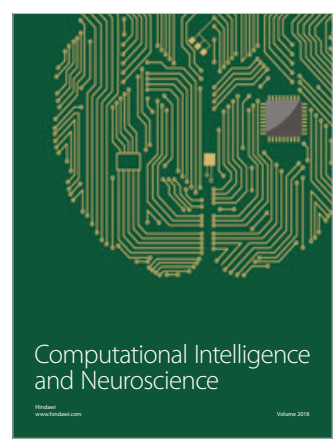

